\title{
Multiple populations in the old and massive Small Magellanic Cloud globular cluster NGC $121^{1}$
}

\author{
E. Dalessandro ${ }^{2,3}$, E. Lapenna ${ }^{2,3}$, A. Mucciarelli ${ }^{2,3}$, L. Origlia ${ }^{3}$, F. R. Ferraro ${ }^{2}$, B. Lanzoni ${ }^{2}$ \\ 2 Dipartimento di Fisica \& Astronomia, Università degli Studi di Bologna, viale Berti \\ Pichat 6/2, I-40127 Bologna, Italy \\ ${ }^{3}$ INAF - Osservatorio Astronomico di Bologna, via Ranzani 1, I-40127, Bologna, Italy
}

\begin{abstract}
We used a combination of optical and near-UV Hubble Space Telescope photometry and FLAMES/ESO-VLT high-resolution spectroscopy to characterize the stellar content of the old and massive globular cluster (GC) NGC 121 in the Small Magellanic Cloud (SMC). We report on the detection of multiple stellar populations, the first case in the SMC stellar cluster system. This result enforces the emerging scenario in which the presence of multiple stellar populations is a distinctive-feature of old and massive GCs regardless of the environment, as far as the light element distribution is concerned. We find that second population (SG) stars are more centrally concentrated than first (FG) ones. More interestingly, at odds with what typically observed in Galactic GCs, we find that NGC 121 is the only cluster so far to be dominated by FG stars that account for more than $65 \%$ of the total cluster mass. In the framework where GCs were born with a $90-95 \%$ of FG stars, this observational finding would suggest that either NGC 121 experienced a milder stellar mass-loss with respect to Galactic GCs or it formed a smaller fraction of SG stars.
\end{abstract}

Subject headings: Magellanic Clouds - globular clusters: individual (NGC 121) - stars: abundances - stars: RGB - techniques: photometric, spectroscopic

\footnotetext{
${ }^{1}$ Based on observations collected with the NASA/ESA HST, obtained at the Space Telescope Science Institute, which is operated by AURA, Inc., under NASA contract NAS5-26555 and collected at the ESOVLT under the program 086.D-0665.
} 


\section{Introduction}

All relatively massive $\left(>4-5 \times 10^{4} M_{\odot}\right)$ and old $(10-13$ Gyr $)$ Galactic globular clusters (GCs) studied so far host multiple stellar populations showing appreciable differences in the abundance of $\mathrm{He}$ and several other light elements (e.g., C, N, Na, O, Al, Mg, see Gratton et al. 2012, for a review). Hereafter, we define these multiple populations as light element MPs (LE-MPs), to distinguish them from the multiple populations showing large iron abundance differences, as those observed in $\omega$ Cen (see e.g. Pancino et al. 2000; Ferraro et al. 2004; Johnson et al. 2010) and in Terzan 5 (Ferraro et al. 2009; Massari et al. 2012; Origlia et al. 2013). These LE-MPs manifest themselves as multi-modal or broadened evolutionary sequences in color-magnitude-diagrams (CMDs), when appropriate filters (or filter combinations) are adopted (see, for example, Piotto et al. 2007; Marino et al. 2009; Monelli et al. 2013; Dalessandro et al. 2011a, 2014a; Milone et al. 2013, 2015).

Different scenarios have been proposed over the years to explain the formation of LEMPs. In the most popular formation models, LE-MPs are the result of a self-enrichment process, which likely occurred in the very early epochs of GC evolution ( $100 \mathrm{Myr})$. A second generation (SG) formed from a combination of the ejecta of stars from a first population (polluters - FG) and from a "pristine material" (Decressin et al. 2007; D'Ercole et al. 2008; de Mink et al. 2009; Conroy 2012; Denissenkov \& Hartwick 2014). However, all of the proposed scenarios face serious problems as none can explain more than few relevant observations (e.g. Salaris \& Cassisi 2014; Bastian \& Lardo 2015; Renzini et al. 2015).

As a matter of fact we still lack a self-consistent explanation of the physical process(es) at the basis of the multiple population formation and evolution. To this end it is necessary to use a thorough and wide approach that combines photometric, spectroscopic and kinematical information. For example, crucial insights on the very first epoch of GC evolution can be obtained by observing and comparing clusters over a wide range of properties (metallicities, mass, structural parameters etc.) and in different environments (from dwarf to giant elliptical galaxies). One of the most debated and still poorly understood topics is what determines the fraction of SG stars in GCs.

By using the available collection of spectroscopic and photometric data, Bastian \& Lardo (2015) showed that the fraction of SG stars is remarkably uniform $\left(N_{\mathrm{SG}} / N_{\mathrm{TOT}} \sim 0.68 \pm 0.07\right)$ regardless of the GC mass, metallicity, and present-day galactocentric distance, with the only exceptions of NGC 6362 (Dalessandro et al. 2014a; Mucciarelli et al. 2016) and NGC5272 (Massari et al. 2016) that show equally-populated FG and SG. To reproduce this evidence, models require that all GCs had an initial mass up to 10-100 times larger than the current one, and they eventually lost $>90-95 \%$ of their original mass through tidal-stripping or gas-expulsion (D'Ercole et al. 2008; Conroy 2012). This strong requirement, which is 
common to any model, is typically known as the "mass-budget problem" and it may have important implications on the galaxy mass-assembly scenarios. However, it should be also noted that it appears to be inconsistent with some observational results (e.g. Larsen et al. 2012, Bastian \& Lardo 2015) and GC dynamical evolution models (e.g. Kruijssen 2015).

On the same line, important efforts have been made to detect LE-MPs in other galaxies. The presence of LE-MPs has been directly observed by means of photometry and/or spectroscopy of resolved stars in the old GCs of the Large Magellanic Clouds (see, for ex-

ample, Mucciarelli et al. 2009a) and in the Fornax dwarf galaxy (Larsen et al. 2014), while it has been indirectly inferred in the massive GCs of M31 (Schiavon et al. 2013) and M87 (Chung et al. 2011) thanks to integrated spectro-photometric studies. Quite surprisingly, similar investigations still lack for the relatively close $(d \sim 60 \mathrm{kpc})$ Small Magellanic Cloud (SMC) irregular dwarf galaxy.

The SMC harbors a population of GCs with an almost continuous distribution in age up to $t \sim 8$ Gyr (Harris \& Zaritsky 2004; Carrera et al. 2008; Dias et al. 2010). The only significant exception is the older cluster NGC 121 , a system located at $\sim 2.3^{\circ} \mathrm{NW}$ from the optical center of the SMC (Crowl et al. 2001). It has a mass $M \simeq 3 \times 10^{5} M_{\odot}$ (Mackey \& Gilmore 2003) and age $t_{\mathrm{AGE}} \sim 11 \mathrm{Gyr}$ (Glatt et al. 2008). These properties make NGC 121 an extremely interesting target, as it is the only old, Galactic-like GC in the SMC.

In this work we present a spectro-photometric analysis of NGC 121 with the aim of characterizing its light element chemical patterns, the presence of LE-MPs (as traced by C, $\mathrm{N}, \mathrm{O}, \mathrm{N}, \mathrm{Mg}$ and $\mathrm{Al}$ abundances) and their properties. The paper is structured as follows: in Section 2 the observational data-base is presented, in Sections 3 and 4 the results obtained by means of the photometric and spectroscopic data-sets, respectively, are discussed. In Section 5 we summarize the most relevant results and draw our conclusions.

\section{Observations and data reduction}

In order to investigate the possible presence of LE-MPs in NGC 121, we analyzed HST high-resolution photometric data and some high-resolution UVES-FLAMES spectra.

\subsection{Analysis of the photometric data-set}

The photometric data-base used in this work consists of a combination of HST images obtained with the Wide Field Camera 3/Ultraviolet and Visible Channel (WFC3/UVIS). 
The WFC3/UVIS data-set (Prop: 13435, PI: Monelli) consists of 10 images, four exposures with $t_{\exp }=200 \mathrm{~s}$ acquired through the $F 438 \mathrm{~W}$ band, four with $t_{\exp }=1061 \mathrm{~s}$ acquired through the $F 336 W$ and two images in the $F 814 W$ band with $t_{\exp }=100 \mathrm{~s}$. The cluster core is centered in chip\#2 in a sub-sample of five images $(2 \times F 438 W, 2 \times F 336 W$ and $1 \times F 814 W)$, and in chip\# 1 in the remaining five. The two sub-groups of images are rotated by $\sim 30^{\circ}$.

The images have been processed, flat-field corrected and bias-subtracted by using standard HST pipelines (_flt images). Pixel-area effects have been accounted for by applying the most updated pixel-area-maps (PAM images) to each image by means of IRAF tasks 2 .

The photometric analysis has been performed independently on each chip by using DAOPHOTIV (Stetson 1987). For each image we selected several tens of bright and relatively isolated stars to model the point spread function (PSF). We used a Moffat analytic function and a second-order spatial variation for the PSF. A first star list has been obtained for each image by independently fitting all the star-like sources, as detected by using a relatively shallow threshold at $6 \sigma$ from the local background. We then used ALLFRAME (Stetson 1994) on all the WFC3/UVIS images, starting from an input list of stars detected in at least four WFC3/UVIS images.

The final star-lists obtained for each image and chip have been then crossed-matched by using DAOMATCH. For each filter, single magnitude estimates were homogenized and their weighted mean and standard deviation were finally calculated by means of DAOMASTER. The final catalog consists of stars measured in the three bands.

To independently assess the quality and completeness of the obtained photometry in the innermost and densest regions of NGC 121, we complemented the WFC3/UVIS data with archival Advanced Camera for Survery/High Resolution Channel (ACS/HRC) data. The ACS/HRC set (Prop: 10369, PI: Gallagher) consists of 12 images, six obtained in the $F 555 \mathrm{~W}$ band, four with $t_{\exp }=300 \mathrm{~s}$ and two with $t_{\exp }=25 \mathrm{~s}$, and six $F 814 W$ images, four with $t_{\exp }=260 \mathrm{sec}$ and two short-exposures with $t_{\exp }=20 \mathrm{~s}$. These images homogeneously cover the innermost $\sim 20^{\prime \prime}$ of the cluster. To exploit the improved PSF sampling provided by the ACS/HRC data, in the region where the ACS/HRC and WFC3/UVIS images overlap, we forced the positions of stars identified in at least five ACS/HRC images to be fitted in the WFC3/UVIS ones (see, for example, Dalessandro et al. 2014b). We verified that the results obtained with both approaches are comparable, therefore for clarity and sake of homogeneity

\footnotetext{
${ }^{2}$ IRAF is distributed by the National Optical Astronomy Observatory, which is operated by the Association of Universities for Research in Astronomy, Inc., under cooperative agreement with the National Science Foundation
} 
in the following we will use only the WFC3/UVIS catalog.

Instrumental magnitudes have been converted to the VEGAMAG photometric system by using the prescriptions and zero-points reported on the dedicated HST web-pages 3 . The instrumental positions of stars have been separately roto-translated to the absolute $(\alpha, \delta)$ coordinates by using as secondary astrometric standards, calibrated onto the 2MASS system, the stars in common with our own near infrared SOFI/NTT photometric catalog (this is part of the same survey presented in Mucciarelli et al. 2009b; we refer to that paper for details about the photometric analysis).

\subsection{Analysis of the spectroscopic data-set}

Five giant stars located at a radial distance of $20^{\prime \prime}<r<40^{\prime \prime}$ from the cluster center (Glatt et al. 2008) have been observed with UVES-FLAMES (Pasquini et al. 2002) under the program 086.D-0665 (PI: Mucciarelli) using the grating 580 Red Arm CD\#3, which provides a high spectral resolution $(\mathrm{R} \sim 40000)$ and a spectral coverage between 4800 and $6800 \AA$. The target stars have been selected from our own near-infrared SOFI/NTT photometric catalog in the brighter portion of the RGB $\left(K_{s} \sim 13-14\right)$, and considering only targets without close companions with comparable or brighter magnitude.

The spectra have been acquired in a series of 13 exposures of $\sim 45 \mathrm{~min}$ each. The data reduction was performed by using the dedicated ESO pipeline including bias subtraction, flat fielding, wavelength calibration, spectral extraction and order merging. Single spectra of each target have been finally co-added, reaching a $\mathrm{S} / \mathrm{N}$ per pixel of $\sim 30$ at $\sim 6000 \AA$. Identification number, right ascension, declination, $J, H, K_{s}$ magnitudes and distance from the cluster centre of the observed stars are listed in Table 1.

The radial velocity of the targets has been determined with DAOSPEC (Stetson \& Pancino 2008) through the measure of about 200 metallic lines per star. The average radial velocity obtained for NGC 121 is $v_{r}=+144.8 \mathrm{~km} \mathrm{~s}^{-1}\left(\sigma=2.0 \mathrm{~km} \mathrm{~s}^{-1}\right)$, in good agreement with previous determinations. In particular, from the analysis of integrated light spectra of NGC 121 Zinn \& West (1984) found $v_{r}=+139 \pm 20 \mathrm{~km} \mathrm{~s}^{-1}$, Hesser et al. (1986) $v_{r}=+138 \pm 15$ $\mathrm{km} \mathrm{s}^{-1}$ and Dubath et al. (1997) $v_{r}=+147 \pm 2 \mathrm{~km} \mathrm{~s}^{-1}$ while Da Costa \& Hatzidimitriou (1998) found $v_{r}=+138 \pm 4 \mathrm{~km} \mathrm{~s}^{-1}$ by using CaII triplet. Based on the measured radial velocities, the five stars are cluster members.

Effective temperatures $\left(\mathrm{T}_{\text {eff }}\right)$ and surface gravities $(\log g)$ have been calculated by using

\footnotetext{
${ }^{3}$ http://www.stsci.edu/hst/wfc3/analysis/uvis_zpts
} 
the photometric information. $\mathrm{T}_{\text {eff }}$ have been derived from the $(J-K)_{0}-\mathrm{T}_{\text {eff }}$ calibration of Alonso et al. (1999), assuming $E(B-V)=0.03$ (Schlegel et al. 1998; Schlafly \& Finkbeiner 2011) and extinction coefficients from (McCall 2004) for the 2MASS photometric system. Surface gravities have been obtained with the Stefan-Boltzmann equation by adopting $(m-$ $M)_{0}=19.02 \mathrm{mag}$ (Glatt et al. 2008). Bolometric corrections were computed following the prescriptions by Buzzoni et al. (2010) and adopting an average stellar mass of $0.8 M_{\odot}$, obtained from a BaSTI isochrone (Pietrinferni et al. 2004) with an age of 11 Gyr and a metallicity $\mathrm{Z}=0.002$, in agreement with the metallicity estimated in this work (see Section 4). Finally, the microturbulent velocities have been derived by requiring no trend between FeI abundances and the line strengths.

The chemical abundances of $\mathrm{Fe}, \mathrm{Na}$ and $\mathrm{Mg}$ have been calculated from the measured equivalent widths (EW) by using the package GALA (Mucciarelli et al. 2013a). The measurements of EWs have been obtained through DAOSPEC, iteratively launched by the 4DAd5 software (Mucciarelli 2013b). We have build a specific linelist for the target stars by using suitable synthetic spectra computed at the UVES resolution and using as guess parameters the photometric estimates. The lines have been carefully selected in order to avoid possible blends and adopting the atomic data of the last release of the Kurucz/Castelli compilation 6. Suitable model atmospheres have been obtained by running the ATLAS97 code and then used as input in the computation of the synthetic spectra with the SYNTHE package (Sbordone et al. 2005).

The Fe abundances have been obtained measuring 100 Fe I lines, the abundances of $\mathrm{Na}$ have been obtained from the lines at 5682, 5688 and $5889 \AA$ and those of $\mathrm{Mg}$ I from the transition at $5711 \AA$. In order to take into account non-local thermodynamical equilibrium effects the Na abundances have been corrected according to Gratton et al. (1999).

We used the spectral synthesis technique, employing the package SALVADOR (Mucciarelli et al., in prep), which follows the method already described in Mucciarelli et al. (2012) to derive the abundances of $\mathrm{O}$ (because the forbidden oxygen line at $6300.3 \AA$ is contaminated by a close $\mathrm{Ni}$ line) and to estimate upper limits for the abundances of $\mathrm{Al}$ (because the $\mathrm{Al}$ lines at 6696-6698 $\AA$ are too weak to be properly measured). We have checked that the [OI] line is free from telluric contamination and we adopted a proper $\mathrm{C}$ and $\mathrm{N}$ abundances

\footnotetext{
${ }^{4}$ http://www.cosmic-lab.eu/gala/gala.php

${ }^{5}$ http://www.cosmic-lab.eu/4dao/4dao.php

${ }^{6}$ http://www.user.oats.inaf.it/castelli/linelists.html

${ }^{7}$ http://www.user.oats.inaf.it/castelli/sources/atlas9codes.html
} 
according to the estimate of Gratton et al. (2000) for RGB stars close to the tip. Finally, we adopted the solar reference values of Grevesse \& Sauval (1998) for Na, Al, Mg and Fe and of Caffau et al. (2011) for O. Table 2 lists the atmospheric parameters and the derived iron abundances, while Table 3 lists some abundance ratios of interest.

For the computation of internal uncertainties two main sources of error have been considered:

(1) the error arising from the EW measurements has been computed by dividing the line-to-line dispersion by the square root of the number of lines used. Thanks to the high number of available lines for FeI we obtained internal uncertainties lower than 0.02 dex. For the $\mathrm{Na}$ abundances we obtained uncertainties in the range 0.03-0.06 dex. For Mg abundances, based on only one line, we assumed as abundance uncertainties those obtained from the EW errors provided by DAOSPEC. For O abundances, obtained through spectral synthesis, we resorted to Monte Carlo simulations following the method described by Mucciarelli et al. (2013c).

(2) the error arising from atmospheric parameters has been estimated by taking into account the photometric errors, which yield an average uncertainty for $\mathrm{T}_{\text {eff }}$ of $\sim 100 \mathrm{~K}$. This value translates in an uncertainty on $\log g$ of $\sim 0.05$ dex. According to these values, we finally estimated that a reasonable uncertainty for the microturbulence is $0.1 \mathrm{~km} \mathrm{~s}^{-1}$. The typical final uncertainties have been obtained by varying each parameter independently and the cumulative one was computed by summing in quadrature the different terms. We found $\sigma_{\mathrm{Fe}}=0.09 \operatorname{dex}, \sigma_{\mathrm{O}}=0.03 \operatorname{dex}, \sigma_{\mathrm{Na}}=0.1 \mathrm{dex}$, and $\sigma_{\mathrm{Mg}}=0.08$ dex.

\section{Results from the photometric data-set}

The $\left(m_{\mathrm{F} 438 \mathrm{~W}}, m_{\mathrm{F} 438 \mathrm{~W}}-m_{\mathrm{F} 814 \mathrm{~W}}\right)$ CMD of NGC121 obtained with the WFC3/UVIS data is shown in Figure 1. All sequences are well defined and they appear to be properly sampled from the RGB-tip to $\sim 2$ magnitudes below the MS turnoff. As expected, given both the position of NGC 121 in the SMC and the fact that we are sampling only the innermost regions of the cluster, very little contamination from field stars appears in the CMD as a sparse sequence of young MS stars overlapping the cluster's Blue Straggler star population.

To search for the presence of LE-MPs we analyzed the ( $\left.m_{\mathrm{F} 336 \mathrm{~W}}, m_{\mathrm{F} 336 \mathrm{~W}}-m_{\mathrm{F} 438 \mathrm{~W}}\right) \mathrm{CMD}$, which is well known to be sensitive to variations of the $\mathrm{NH}$ and $\mathrm{CN}-\mathrm{CH}$ molecular bands (Sbordone et al. 2011). Indeed, in the CMD of Figure 2, the color broadening of the RGB significantly exceeds the one expected purely from photometric errors, and a hint of sequence split appears quite clearly at $m_{\mathrm{F} 336 \mathrm{~W}}<22$. It has been demonstrated (Marino et al. 2008; 
Sbordone et al. 2011; Dalessandro et al. 2014a; Mucciarelli et al. 2016) that in the $\left(m_{\mathrm{F} 336 \mathrm{~W}}\right.$, $\left.m_{\mathrm{F} 336 \mathrm{~W}}-m_{\mathrm{F} 438 \mathrm{~W}}\right)$ CMD stars enhanced in $\mathrm{N}$ and $\mathrm{Na}$ and depleted in $\mathrm{O}$ and $\mathrm{C}$ (SG) populate the red part of the RGB, while the $\mathrm{Na} / \mathrm{N}$-poor and O/C-rich stars (FG) occupy the blue part.

To maximize the separation between different sub-populations, we used the HST version of the color index $C_{\mathrm{U}, \mathrm{B}, \mathrm{I}}$ (Monelli et al. 2013), defined as $\left(C_{F 336 W, F 438 W, F 814 W}\right)=\left(m_{\mathrm{F} 336 \mathrm{~W}}-\right.$ $\left.m_{\mathrm{F} 438 \mathrm{~W}}\right)-\left(m_{\mathrm{F} 438 \mathrm{~W}}-m_{\mathrm{F} 814 \mathrm{~W}}\right)$. This color combination is sensitive to both light metals and He variations (Sbordone et al. 2011; Monelli et al. 2013) and it has been proven to effectively separate LE-MPs in GCs. The $\left(m_{\mathrm{F} 336 \mathrm{~W}}, C_{F 336 W, F 438 W, F 814 W}\right) \mathrm{CMD}$ is shown in the left panel of Figure 3, In this diagram, for $m_{\mathrm{F} 336 \mathrm{~W}}<22$ the RGB clearly splits in two sequences, with the redder sequence corresponding to O-rich/Na-poor (FG), while the bluer sequence being associated to the O-poor/Na-rich (SG) sub-populations (Monelli et al. 2013).

In order to better distinguish the sub-populations, for RGB stars in the magnitude range $20.45<m_{\mathrm{F} 336 \mathrm{~W}}<22.00$ we computed the distribution of the color distance from the RGB mean ridge line $8\left(\Delta C_{F 336 W, F 438 W, F 814 W}\right.$, hereafter $\left.\Delta C\right)$ in the $\left(m_{\mathrm{F} 336 \mathrm{~W}}, C_{F 336 W, F 438 W, F 814 W}\right)$ CMD (Figure 3, right panels). The distribution is clearly bimodal. To test the statistical significance of the bimodality, we performed the Gaussian Mixture Modeling (GMM) test (Muratov \& Gnedin 2010) finding that, as expected, the hypothesis of unimodality can be rejected with a probability $>99.9 \%$. The distribution is well fitted by two Gaussians with peaks at $\Delta C=-0.055 \pm 0.004 \mathrm{mag}$ and $\Delta C=+0.037 \pm 0.005 \mathrm{mag}$ and both with a dispersion $\sigma \sim 0.025$, which is compatible with the color errors in the considered magnitude range. We classified all stars with values of $\Delta C$ larger or smaller than 0 as FG and SG sub-populations respectively. With this selection we count 457 FG and 247 SG stars in the WFC3/UVIS field of view, corresponding to the $65 \pm 4 \%$ and $35 \pm 3 \%$ of the total. At odds with what typically found so far in Galactic GCs, NGC 121 is dominated by FG stars. It is important to note also that, as the HST field of view ensures a sampling of $\sim 70 \%$ of the entire cluster extension $\left(r_{t} \sim 165^{\prime \prime}\right.$, Glatt et al. 2009), we can consider these population ratios as a good approximation of the global ones.

We estimated the RGB width $\left(W_{R G B}\right)$, which is defined as the extension of the $\Delta C$ distribution, after removing the 5 per cent bluest and reddest stars. We found $W_{R G B} \sim 0.16$ mag, which turns out to be slightly larger than what previously obtained for clusters with a similar metallicity (see the case of NGC 288 which has $W_{R G B}=0.14$ mag; Monelli et al.

\footnotetext{
${ }^{8}$ To derive the RGB mean ridge line we considered only as sub-sample of "fiducial" RGB stars defined as those falling in the hand-drawn box in the left panel of Figure 3 . We then computed the median color of RGB stars in bins of 0.3 magnitudes and interpolated them with a spline.
} 
2013). Given the almost linear correlation between the $[\mathrm{Na} / \mathrm{Fe}]$ and $\Delta C$ distributions found by Monelli et al. (2013), this observational finding would suggest a wider $[\mathrm{Na} / \mathrm{Fe}]$ dispersion in NGC 121 than in Galactic GCs with similar metallicity where this analysis has been performed.

To asses the completeness of our catalog we performed extensive artificial star experiments following the approach described in Dalessandro et al. (2011b; 2015). Briefly, we first derived mean ridge lines in the $\left(m_{\mathrm{F} 336 \mathrm{~W}}, m_{\mathrm{F} 336 \mathrm{~W}}-m_{\mathrm{F} 438 \mathrm{~W}}\right)$ and $\left(m_{\mathrm{F} 336 \mathrm{~W}}, m_{\mathrm{F} 336 \mathrm{~W}}-m_{\mathrm{F} 814 \mathrm{~W}}\right)$ CMD, adopting 0.5 mag-wide bins in $m_{\mathrm{F} 336 \mathrm{~W}}$ and selecting the corresponding median values obtained after a $2 \sigma$-clipping rejection. Then we generated a catalog of simulated stars with a $m_{\mathrm{F} 336 \mathrm{~W}}$-input magnitude extracted from a luminosity function modeled to reproduce the observed one. To each star extracted we assigned input magnitudes $m_{\mathrm{F} 438 \mathrm{~W}}^{i n}$ and $m_{\mathrm{F} 814 \mathrm{~W}}^{i n}$ by means of an interpolation along the mean ridge line. To prevent any artificial increase of the crowding conditions, we simulated only one artificial star within a $25 \times 25$ pixel box at each run. This procedure was performed by using the same PSF models and reduction procedures described in Section 2.1. The artificial star catalog thus obtained was used to derive the completeness curves as the ratio $C=N_{\text {out }} / N_{\text {in }}$ between the number of stars recovered after the photometric reduction $\left(N_{\text {out }}\right)$ and the number of simulated stars $\left(N_{\text {in }}\right)$ in each magnitude bin. The completeness curves as a function of the $m_{\mathrm{F} 336 \mathrm{~W}}$ magnitude at different distances from the cluster center are shown in Figure 4. In the range of magnitude where LE-MPs have been identified the completeness $C \sim 100 \%$.

The photometric data-set allows to sample the cluster area within $\sim 120^{\prime \prime}$, corresponding to $\sim 4.5$ half-light radii $\left(r_{h}=27.1^{\prime \prime}\right.$, Glatt et al. 2008). The cumulative radial distribution of the FG and SG sub-populations is shown in Fig. 5. It clearly reveals that SG stars are significantly more centrally concentrated than the FG sub-population, as assessed by means of a Kolmogorov-Smirnov test. This is in agreement with what observed in most Galactic GCs studied so far, apart from NGC 6362 (Dalessandro et al. 2014a), NGC 6752 and NGC 6121 (Nardiello et al. 2015), where FG and SG are totally spatially mixed, and M 15 (Larsen et al. 2015), where FG have been suggested to be more segregated than SG stars.

The radial distribution of the ratio $N_{\mathrm{SG}} / N_{\text {TOT }}$ between $\mathrm{SG}$ and the total number of stars (Figure 5, bottom panel) indicates that SG stars are more centrally concentrated, showing a maximum $N_{\mathrm{SG}} / N_{\mathrm{TOT}} \sim 0.45$ at $r<10^{\prime \prime}$ and progressively decreasing values moving outward, down to a minimum value, $N_{\mathrm{SG}} / N_{\mathrm{TOT}} \sim 0.10$ at $r \sim 60^{\prime \prime}$. 


\section{Results from the spectroscopic data-set}

The chemical analysis of NGC 121 provides an average iron abundance $[\mathrm{Fe} / \mathrm{H}]=-1.28$ $\operatorname{dex}(\sigma=0.06 \mathrm{dex})$, with no hint of intrinsic iron spread. This value is higher than previous derivations by Mighell et al. (1998) who found $[\mathrm{Fe} / \mathrm{H}]=-1.71 \mathrm{dex}$, Da Costa \& Hatzidimitriou (1998) $[\mathrm{Fe} / \mathrm{H}]=-1.51 \mathrm{dex}$, Zinn \& West (1984) $[\mathrm{Fe} / \mathrm{H}]=-1.46 \mathrm{dex}$, and Johnson et al. (2004) $[\mathrm{Fe} / \mathrm{H}]=-1.41$. However, these analysis were based on photometry or low-resolution spectra that are significantly more prone to uncertainties and to systematics related to the adopted calibrations.

No intrinsic spreads have been found also for light-element abundances $[\mathrm{O} / \mathrm{Fe}],[\mathrm{Na} / \mathrm{Fe}]$ and $[\mathrm{Mg} / \mathrm{Fe}]$, as confirmed using the Maximum Likelihood algorithm described in Mucciarelli et al. (2012) that rules out any significant light-element spread in the sample.

Figure 6 shows the location of the five RGB stars measured in NGC 121 (blue circles) in the $[\mathrm{O} / \mathrm{Fe}]-[\mathrm{Na} / \mathrm{Fe}]$ and $[\mathrm{Mg} / \mathrm{Fe}]-[\mathrm{Al} / \mathrm{Fe}]$ diagrams, in comparison with the individual stars measured in the Galactic GCs (gray points, data from Carretta et al. 2009a, b) and in the old LMC GCs (red squares, Mucciarelli et al. 2009a). At variance with the stars in the Galactic and LMC old GCs, that share the same mean loci and cover a large range in the light-element abundances, the stars of NGC 121 cover a small area in the two planes shown in Figure 6, pointing out that these stars are indeed internally homogeneous. At a first order approximation, the position of these stars in both the $[\mathrm{O} / \mathrm{Fe}]-[\mathrm{Na} / \mathrm{Fe}]$ and $[\mathrm{Mg} / \mathrm{Fe}]-[\mathrm{Al} / \mathrm{Fe}]$ diagrams is consistent with that of Galactic and LMC old globular FG stars. We note however that they do not follow exactly the distribution of MW and LMC GCs, especially in the $[\mathrm{Mg} / \mathrm{Fe}]-[\mathrm{Al} / \mathrm{Fe}]$ diagram. This might be related to the different chemical evolution history of the SMC.

This finding suggests that the observed spectroscopic sample is composed only by FG stars. Although the analyzed sample is admittedly small, clear evidence of light-element spread and some anti-correlations were found by Mucciarelli et al. (2009a) in old LMC clusters, based on a similar number of stars (5-7). However the lack of SG stars in the spectroscopic sample of NGC 121 is qualitatively compatible with the fact this is dominated by FG stars that account for $\sim 70 \%$ of the total population at the surveyed distances.

\section{Discussion and Conclusions}

The spectro-photometric analysis of the old and massive SMC cluster NGC 121 performed in this paper, provided the following main results: 
- NGC 121 shows a clear broadening and/or splitting of the RGB sequence in CMDs where the F336W filter is used (Section 3, Figures 2 and 3). This result represents the first evidence that NGC 121 hosts LE-MPs. This cluster is also the first system to show such a property in the SMC, thus enforcing the emerging scenario in which LE-MPs are a distinctive-feature of old and massive GCs, regardless of the environment. In fact, this feature is observed in the Milky Way, in the Fornax dwarf galaxy and, now, in both the Magellanic Clouds.

- SG stars are more centrally concentrated than FG stars, as typically observed in Galactic GCs (Section 3, Figure 5).

- NGC 121 is dominated by FG stars that account for $65 \%$ of the entire population of the cluster, at variance with what found in Galactic GCs, where the fraction of FG stars is smaller than the SG one.

- The analysis of high-resolution spectra of five RGB stars yields an average iron abundance $[\mathrm{Fe} / \mathrm{H}]=-1.28$ dex $(\sigma=0.06 \mathrm{dex})$, with no hints of intrinsic iron spread (Section (4). Homogeneous light-element abundance ratios $([\mathrm{O} / \mathrm{Fe}],[\mathrm{Na} / \mathrm{Fe}]$ and $[\mathrm{Mg} / \mathrm{Fe}]-$ Figure 6) have been also found, consistent with all the analyzed stars being O-rich/Napoor FG stars.

D'Ercole et al. (2008) and Vesperini et al. (2013) suggested that at birth GCs should be populated by $\sim 95 \%$ of FG stars. Then they reach present-day number ratios because of a preferential FG star loss due to both early stellar $(t<1-2 \mathrm{Gyr})$ and long-term dynamical evolutions.

Figure 7 shows the location of NGC 121 in the Mass vs $N_{\mathrm{SG}} / N_{\mathrm{TOT}}$ diagram, together with the values measured in NGC 6362 (Dalessandro et al. 2014a; Mucciarelli et al. 2016) and NGC 5272 (Massari et al. 2016), by using the same photometric technique to distinguish among different sub-populations. For comparison we also show the average $N_{\mathrm{SG}} / N_{\mathrm{TO}}$ ratio computed by Bastian \& Lardo (2015) using a collection of spectroscopic and photometric measurements for 33 Galactic GCs, which stays constant at a value of (68 $\pm 7 \%$ ).

It is worth noticing that the SG fraction observed in NGC 121 appears to be consistent with the value predicted by the gas-expulsion model of Khalaj \& Baumgardt (2015) based on N-body simulations (Figure 7), which foresees a decreasing SG star fraction with increasing the cluster mass. Such a similarity is quite remarkable, as at the mass of NGC 121, these models underpredict by a factor of three or more the fraction of SG stars in Galactic GCs (Figure 7). 
A dominant FG sub-population in NGC 121 can be explained with a less severe mass loss (still $\sim 90 \%$ ) experienced by this cluster, when compared to Galactic GCs, due to weak tidal interactions with the intrinsically shallower potential of the SMC, and to the fact that this $\mathrm{GC}$ orbits at a quite large distance (about $2.5 \mathrm{kpc}$ ) from the SMC center. Another possible explanation is that NGC 121 lost the same fraction of FG stars as Galactic GCs, but formed a smaller fraction of SG stars, because of, e.g., a less efficient self-enrichment processes, gas retention, the impact of different environments on LE-MP formation, etc.

However, on top of these speculative arguments, it should be noted that the available FG and SG population ratios for Galactic GCs can be quite uncertain and not well representative of the entire cluster population. In fact, spectroscopic-based number ratios strongly depend on the the ability to separate different sub-populations that sometimes have not discretely different abundance patterns. Moreover, they are spatially incomplete by construction, typically not sampling the cluster central regions. On the other hand, photometric derivations are generally limited to the small HST field of view and cannot account for possible largescale radial variations.

Such potential biases in the derived population ratios in most of the Galactic GCs are a serious concern. Indeed, robust estimates obtained in NGC 6362 (Figure 7; Dalessandro et al. 2014a) and NGC 5272 (Massari et al. 2016), for which global number ratios are available (thanks to a proper combination of HST and ground-based wide-field observations), indicate $N_{\mathrm{SG}} / N_{\text {TOT }} \sim 50 \%$, significantly lower than the average value of $\sim 0.7$ computed by Bastian \& Lardo (2015).

Concluding, NGC 121 turns out to be an interesting and intriguing case among the old, massive GCs studied so far. Understanding the origin of its peculiarities, will likely provide important insights into the formation and early evolution of GCs and multiple populations. Moreover, performing similar studies on a larger sample of clusters will allow us to shed new light on the role of the environment and host galaxy properties on the multiple population properties.

We thank the anonymous referee for his/her comments and suggestions. L.O. and E.L. acknowledge the PRIN-INAF 2014 CRA 1.05.01.94.11: "Probing the internal dynamics of globular clusters. The first comprehensive radial mapping of individual star kinematics with the new generation of multi-object spectrographs" (PI: L. Origlia). 


\section{REFERENCES}

Alonso, A., Arribas, S., \& Martínez-Roger, C. 1999, A\&AS, 140, 261

Bastian, N., Lamers, H. J. G. L. M., de Mink, S. E., et al. 2013, MNRAS, 436, 2398

Bastian, N., \& Lardo, C., 2015, MNRAS, 453, 357

Baume, G., Noël, N. E. D., Costa, E., et al. 2008, MNRAS, 390, 1683

Buzzoni, A., Patelli, L., Bellazzini, M., Pecci, F. F., \& Oliva, E. 2010, MNRAS, 403, 1592

Caffau, E., Ludwig, H.-G., Steffen, M., Freytag, B., \& Bonifacio, P. 2011, Sol. Phys., 268, 255

Carrera, R., Gallart, C., Aparicio, A., et al. 2008, AJ, 136, 1039

Carretta, E., Bragaglia, A., Gratton, R. G., et al. 2009a, A\&A, 505, 117

Carretta, E., Bragaglia, A., Gratton, R., \& Lucatello, S. 2009b, A\&A, 505, 139

Chung, C., Yoon, S.-J., \& Lee, Y.-W. 2011, ApJ, 740, L45

Conroy, C. 2012, ApJ, 758, 21

Crowl, H. H., Sarajedini, A., Piatti, A. E., et al. 2001, AJ, 122, 220

Da Costa, G. S., \& Hatzidimitriou, D. 1998, AJ, 115, 1934

Dalessandro, E., Salaris, M., Ferraro, F. R., et al. 2011, MNRAS, 410, 694

Dalessandro, E., Lanzoni, B., Beccari, G., et al. 2011, ApJ, 743, 11

Dalessandro, E., Massari, D., Bellazzini, M., Miocchi, P., Mucciarelli, A., Salaris, M., Cassisi, S., Ferraro, F. R., \& Lanzoni, B., 2014a, ApJ, 791L, 4

Dalessandro, E., Pallanca, C., Ferraro, F. R., et al. 2014b, ApJ, 784, L29

Dalessandro, E., Ferraro, F. R., Massari, D., et al. 2015, ApJ, 810, 40

Decressin, T., Meynet, G., Charbonnel, C., Prantzos, N., \& Ekström, S. 2007, A\&A, 464, 1029

de Mink, S. E., Pols, O. R., Langer, N., \& Izzard, R. G. 2009, A\&A, 507, L1

Denissenkov, P. A., \& Hartwick, F. D. A. 2014, MNRAS, 437, 21 
D'Ercole, A., Vesperini, E., D’Antona, F., McMillan, S. L. W., \& Recchi, S. 2008, MNRAS, 391,825

Dias, B., Coelho, P., Barbuy, B., Kerber, L., \& Idiart, T. 2010, A\&A, 52

Dolphin, A. E., Walker, A. R., Hodge, P. W., et al. 2001, ApJ, 562, 303

Dubath, P., Meylan, G., \& Mayor, M. 1997, A\&A, 324, 505

Ferraro, F. R., Sollima, A., Pancino, E., et al. 2004, ApJ, 603, L81

Ferraro, F.R., et al., 2009, Nature, 462, 483

Glatt, K., Gallagher, J. S., III, Grebel, E. K., et al. 2008, AJ, 135, 1106

Glatt, K., Grebel, E. K., Gallagher, J. S., III, et al., 2009, AJ, 138, 1403

Gratton, R. G., Carretta, E., Eriksson, K., \& Gustafsson, B. 1999, A\&A, 350, 955

Gratton, R. G., Sneden, C., Carretta, E., \& Bragaglia, A. 2000, A\&A, 354, 169

Gratton, R. G., Carretta, E., \& Bragaglia, A. 2012, A\&A Rev., 20, 50

Grevesse, N., \& Sauval, A. J., 1998, Space Science Reviews, 85, 161

Harris, J., \& Zaritsky, D. 2004, AJ, 127, 1531

Hesser, J. E., Shawl, S. J., \& Meyer, J. E. 1986, PASP, 98, 403

Johnson, J. A., Bolte, M., Hesser, J. E., Ivans, I. I., \& Stetson, P. B. 2004, Origin and Evolution of the Elements, 29

Johnson, C. I., \& Pilachowski, C. A., 2010, ApJ, 722, 1373

Khalaj, P., \& Baumgardt, H. 2015, MNRAS, 452, 924

Kruijssen, J. M. D. 2015, MNRAS, 454, 1658

Larsen, S. S., Strader, J., \& Brodie, J. P. 2012, A\&A, 544, L14

Larsen, S. S., Brodie, J. P., Grundahl, F., \& Strader, J. 2014, ApJ, 797, 15

Larsen, S. S., Baumgardt, H., Bastian, N., et al. 2015, ApJ, 804, 71

Mackey, A. D., \& Gilmore, G. F. 2003, MNRAS, 338, 120 
Marino, A. F., Villanova, S., Piotto, G., Milone, A. P., Momany, Y., Bedin, L. R., \& Medling, A. M., 2008, A\&A, 490, 625

Marino, A. F., Milone, A. P., Piotto, G., et al. 2009, A\&A, 505, 1099

Massari, D., Mucciarelli, A., Dalessandro, E., et al. 2012, ApJ, 755, L32

Massari, D., Lapenna, E., Bragaglia, A., et al. 2016, MNRAS, 458, 4162

McCall, M. L. 2004, AJ, 128, 2144

Mighell, K. J., Sarajedini, A., \& French, R. S. 1998, AJ, 116, 2395

Milone, A. P., Marino, A. F., Piotto, G., et al. 2013, ApJ, 767, 120

Milone, A. P., Marino, A. F., Piotto, G., et al. 2015, ApJ, 808, 51

Monelli, M., Milone, A. P., Stetson, P. B., et al. 2013, MNRAS, 431, 2126

Mucciarelli, A., Origlia, L., Ferraro, F. R., \& Pancino, E., 2009, ApJ, 659L, 134

Mucciarelli, A., Origlia, L., Maraston, C., \& Ferraro, F. R. 2009, ApJ, 690, 288

Mucciarelli, A., Bellazzini, M., Ibata, R. et al., 2012, MNRAS, 426, 2889

Mucciarelli, A., Pancino, E., Lovisi, L., Ferraro, F. R., \& Lapenna, E. 2013a, ApJ, 766, 78

Mucciarelli, A. 2013b, arXiv:1311.1403

Mucciarelli, A., Bellazzini, M., Catelan, M., Dalessandro, E., Amigo, P., Correnti, M., Cortés, C., \& D'Orazi, V., 2013c, MNRAS, 435, 3667

Mucciarelli, A., Dalessandro, E., Massari, D., Bellazzini, M., Ferraro, F. R., Lanzoni, B., Lardo, C., Salaris, M., \& Cassisi, S. 2016, ApJ, 824, 73

Muratov, A. L., \& Gnedin, O. Y. 2010, ApJ, 718, 1266

Nardiello, D., Milone, A. P., Piotto, G., et al. 2015, A\&A, 573, A70

Origlia, L., Massari, D., Rich, R. M., Mucciarelli, A., Ferraro, F. R., Dalessandro, E., \& Lanzoni, B. 2013, ApJ, 779, L5

Pancino, E. Ferraro, F. R., Bellazzini, M., PIotto, G., \& Zoccali, M., 2000, ApJ, 534L, 83

Pasquini, L., Avila, G., Blecha, A., et al. 2002, The Messenger, 110, 1 
Pietrinferni, A., Cassisi, S., Salaris, M., \& Castelli, F. 2004, ApJ, 612, 168

Piotto, G., Bedin, L. R., Anderson, J., et al. 2007, ApJ, 661, L53

Renzini, A., D'Antona, F., Cassisi, S., et al. 2015, MNRAS, 454, 4197

Salaris, M., \& Cassisi, S. 2014, A\&A, 566, A109

Sbordone, L., 2005, MSAIS, 8, 61

Sbordone, L., Salaris, M., Weiss, A., \& Cassisi, S., 2011, A\&A, 534, A9

Schiavon, R. P., Caldwell, N., Conroy, C., et al. 2013, ApJ, 776, L7

Schlafly, E. F., \& Finkbeiner, D. P. 2011, ApJ, 737, 103

Schlegel, D. J., Finkbeiner, D. P., \& Davis, M. 1998, ApJ, 500, 525

Shara, M. M., Fall, S. M., Rich, R. M., \& Zurek, D. 1998, ApJ, 508, 570

Shetrone, M. D., Côté, P., \& Sargent, W. L. W. 2001, ApJ, 548, 592

Shetrone, M., Venn, K. A., Tolstoy, E., et al. 2003, AJ, 125, 684

Stryker, L. L., Da Costa, G. S., \& Mould, J. R. 1985, ApJ, 298, 544

Stetson, P. B. 1987, PASP, 99, 191

Stetson, P. B. 1994, PASP, 106, 250

Stetson, P. B., \& Pancino, E., PASP, 120, 1332

Vesperini, E., McMillan, S. L. W., D’Antona, F., \& D'Ercole, A. 2013, MNRAS, 429, 1913

Zinn, R., \& West, M. J. 1984, ApJS, 55, 45

This preprint was prepared with the AAS IATEX macros v5.2. 
Table 1. Identification number, right ascension, declination, J, H, K magnitudes, distance from the cluster center and radial velocity of the target stars.

\begin{tabular}{rccccccc}
\hline \hline ID & $\begin{array}{c}\text { R.A. } \\
(\mathrm{J} 2000)\end{array}$ & $\begin{array}{c}\text { Decl. } \\
(\mathrm{J} 2000)\end{array}$ & $\begin{array}{c}\mathrm{J} \\
(\mathrm{mag})\end{array}$ & $\begin{array}{c}\mathrm{H} \\
(\mathrm{mag})\end{array}$ & $\begin{array}{c}\mathrm{K} \\
(\mathrm{mag})\end{array}$ & $\begin{array}{c}\text { Distance } \\
(\operatorname{arcsec})\end{array}$ & $\begin{array}{c}v_{r} \\
\left(\mathrm{~km} \mathrm{~s}^{-1}\right)\end{array}$ \\
\hline & & & & & & & \\
9 & 6.6842639 & -71.5367593 & 13.977 & 13.262 & 13.111 & 21.7 & $143.90 \pm 0.12$ \\
14 & 6.7033858 & -71.5295107 & 14.253 & 13.560 & 13.440 & 25.8 & $142.17 \pm 0.13$ \\
18 & 6.7233897 & -71.5450034 & 14.371 & 13.677 & 13.552 & 37.7 & $145.25 \pm 0.16$ \\
31 & 6.6845726 & -71.5315588 & 14.671 & 14.024 & 13.966 & 28.2 & $147.91 \pm 0.12$ \\
35 & 6.6970187 & -71.5477942 & 14.716 & 14.048 & 13.934 & 41.0 & $146.23 \pm 0.26$ \\
\hline
\end{tabular}

Note. - The distances have been computed by adopting the cluster center coordinates from Glatt et al. (2008).

Table 2. Atmospheric parameters and $[\mathrm{FeI} / \mathrm{H}]$ abundances for the target stars.

\begin{tabular}{rcccc}
\hline \hline ID & $\begin{array}{c}T_{\text {eff }}^{\text {phot }} \\
(\mathrm{K})\end{array}$ & $\begin{array}{c}\log g^{\text {phot }} \\
(\mathrm{dex})\end{array}$ & $\begin{array}{c}v_{t}^{\text {spec }} \\
\left(\mathrm{km} \mathrm{s}^{-1}\right)\end{array}$ & $\begin{array}{c}{[\mathrm{FeI} / \mathrm{H}]} \\
(\mathrm{dex})\end{array}$ \\
\hline 9 & 3874 & 0.36 & 1.30 & $-1.20 \pm 0.09$ \\
14 & 3904 & 0.48 & 1.45 & $-1.36 \pm 0.09$ \\
18 & 4010 & 0.57 & 1.35 & $-1.28 \pm 0.09$ \\
31 & 4105 & 0.70 & 1.25 & $-1.31 \pm 0.09$ \\
35 & 4159 & 0.77 & 1.25 & $-1.24 \pm 0.09$ \\
\hline
\end{tabular}

Note. - Identification number, photometric temperatures and gravities, microturbulent velocities, and $[\mathrm{FeI} / \mathrm{H}]$ abundance ratios with internal uncertainty. For all the stars a global metallicity of $[\mathrm{M} / \mathrm{H}]=-1.25$ dex has been assumed for the model atmospheres. The reference solar value is taken from Grevesse \& Sauval (1998). 
Table 3. Chemical abundances for light- and $\alpha$-elements of the target stars.

\begin{tabular}{rcccc}
\hline \hline ID & $\begin{array}{c}{[\mathrm{O} / \mathrm{Fe}]} \\
(\mathrm{dex})\end{array}$ & $\begin{array}{c}{[\mathrm{Na} / \mathrm{Fe}]} \\
(\mathrm{dex})\end{array}$ & $\begin{array}{c}{[\mathrm{Mg} / \mathrm{Fe}]} \\
(\mathrm{dex})\end{array}$ & $\begin{array}{c}{[\mathrm{Al} / \mathrm{Fe}]} \\
(\mathrm{dex})\end{array}$ \\
\hline 9 & $0.10 \pm 0.04$ & $-0.66 \pm 0.01$ & $-0.01 \pm 0.08$ & $<-0.17$ \\
14 & $0.28 \pm 0.03$ & $-0.38 \pm 0.06$ & $-0.10 \pm 0.07$ & $<-0.11$ \\
18 & $0.11 \pm 0.05$ & $-0.54 \pm 0.05$ & $-0.21 \pm 0.08$ & $<-0.19$ \\
31 & $0.13 \pm 0.04$ & $-0.40 \pm 0.02$ & $0.11 \pm 0.07$ & $<-0.24$ \\
35 & $0.17 \pm 0.05$ & $-0.50 \pm 0.05$ & $-0.08 \pm 0.08$ & $<-0.33$ \\
\hline
\end{tabular}

Note. - Abundance ratios with internal uncertainty. The abundances of oxygen and magnesium have been derived from one line, while for aluminium we estimated an upper limit only. The reference solar values are taken from Grevesse \& Sauval (1998) for all species except for oxygen for which we adopted the value of Caffau et al. (2011). 


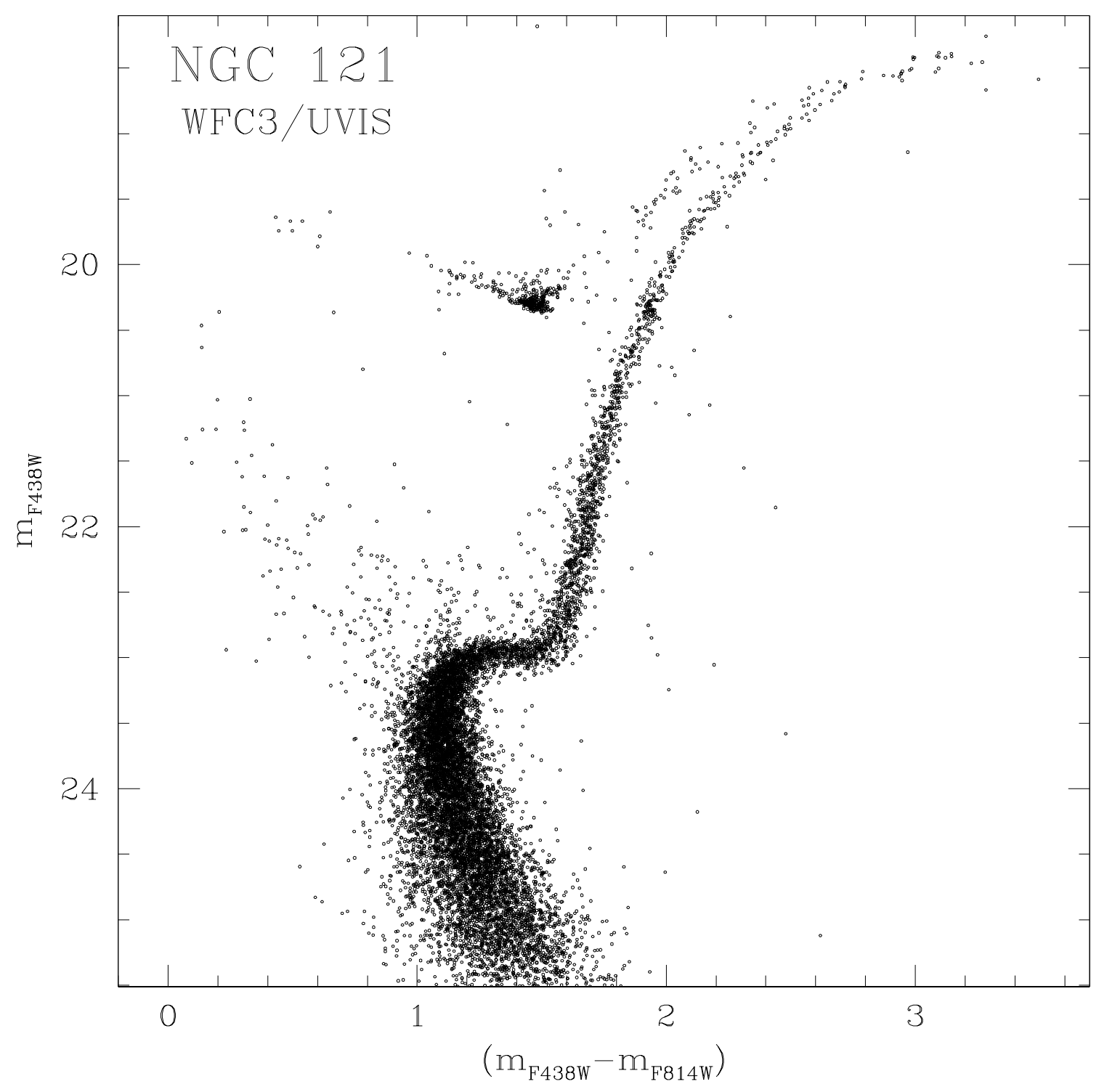

Fig. 1.- $\left(m_{\mathrm{F} 438 \mathrm{~W}}, m_{\mathrm{F} 438 \mathrm{~W}}-m_{\mathrm{F} 814 \mathrm{~W}}\right) \mathrm{CMD}$ of NGC 121 as obtained from the HST WFC3/UVIS data-set. 


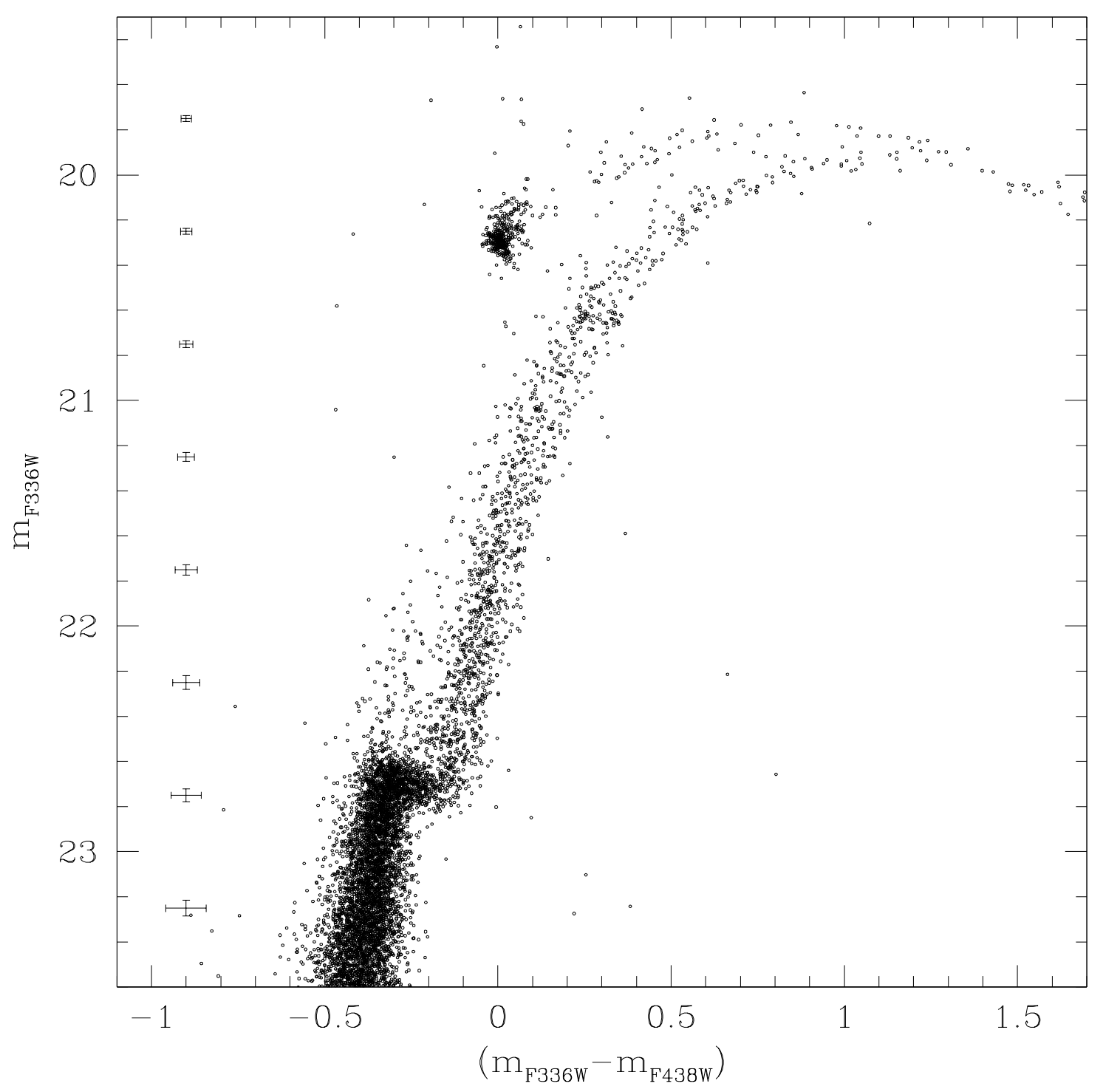

Fig. 2.- $\left(m_{\mathrm{F} 336 \mathrm{~W}}, m_{\mathrm{F} 336 \mathrm{~W}}-m_{\mathrm{F} 438 \mathrm{~W}}\right)$ CMD of NGC 121 as obtained from the HST WFC3/UVIS data-set. The RGB sequence is broader than expected from photometric errors and for $m_{\mathrm{F} 336 \mathrm{~W}}<22$ a hint of splitting is apparent. In this CMD, Na-rich and O-poor SG stars would be located on the red sequence, while Na-poor and O-rich (FG) stars lie on the blue one. 

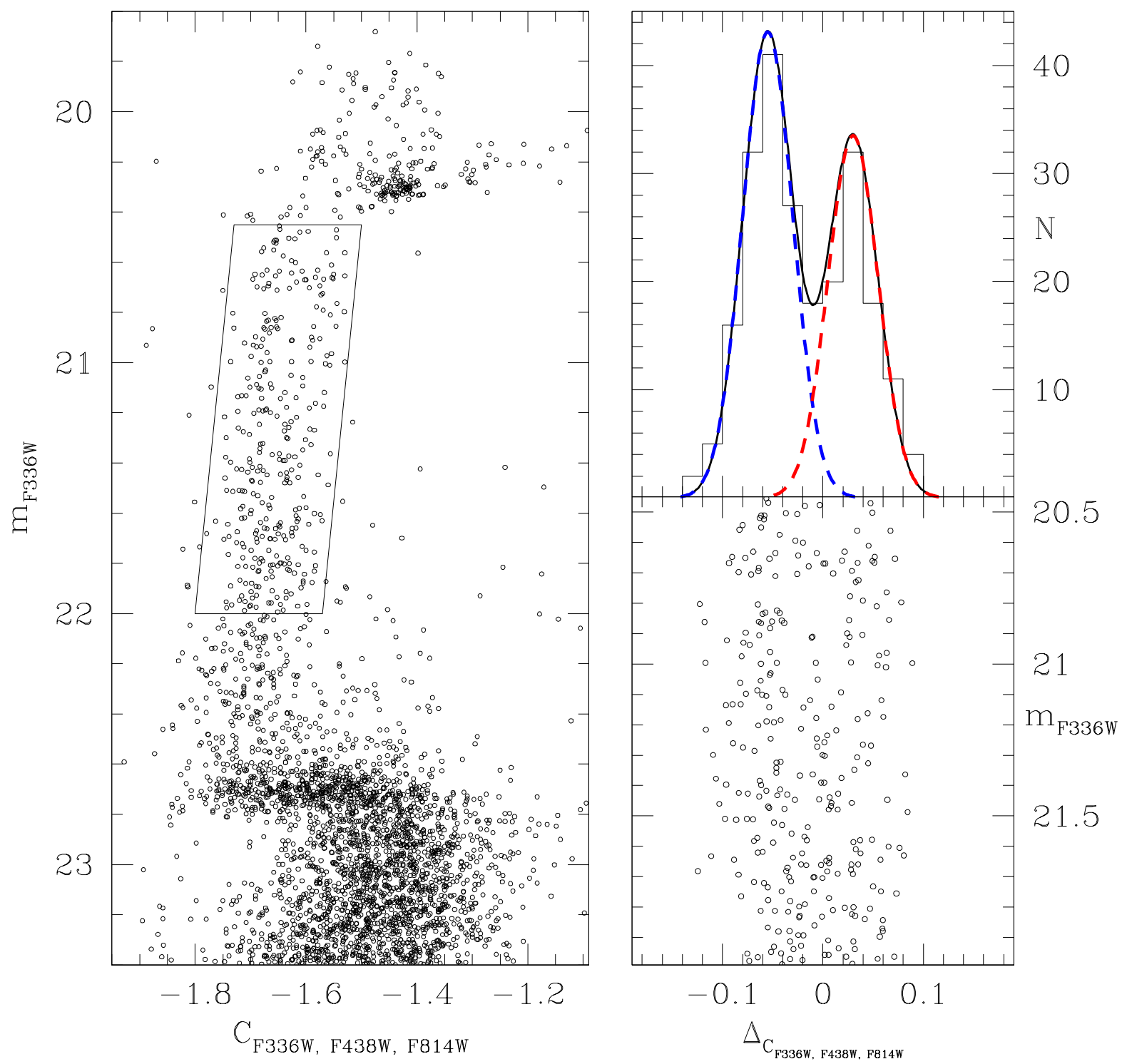

Fig. 3.- Left panel: $\left(m_{\mathrm{F} 336 \mathrm{~W}}, C_{\mathrm{F} 336 \mathrm{~W}, \mathrm{~F} 438 \mathrm{~W}, \mathrm{~F} 814 \mathrm{~W}}\right) \mathrm{CMD}$. The box marks the region where the RGB splitting is more evident and stars have been selected to compute the differential color distribution $\left(\Delta_{\mathrm{C}_{\mathrm{F} 336 \mathrm{~W}, \mathrm{~F} 438 \mathrm{~W}, \mathrm{~F} 814 \mathrm{~W}}}\right)$. Right lower panel: distribution of the color distance $\left(\Delta_{\mathrm{C}_{\mathrm{F} 336 \mathrm{~W}, \mathrm{~F} 438 \mathrm{~W}, \mathrm{~F} 814 \mathrm{~W}}}\right)$ of RGB stars in the magnitude interval $20.45<m_{\mathrm{F} 336 \mathrm{~W}}<22.00$ with respect to the RGB ridge mean line. Right upper panel: histogram of the color distribution. 


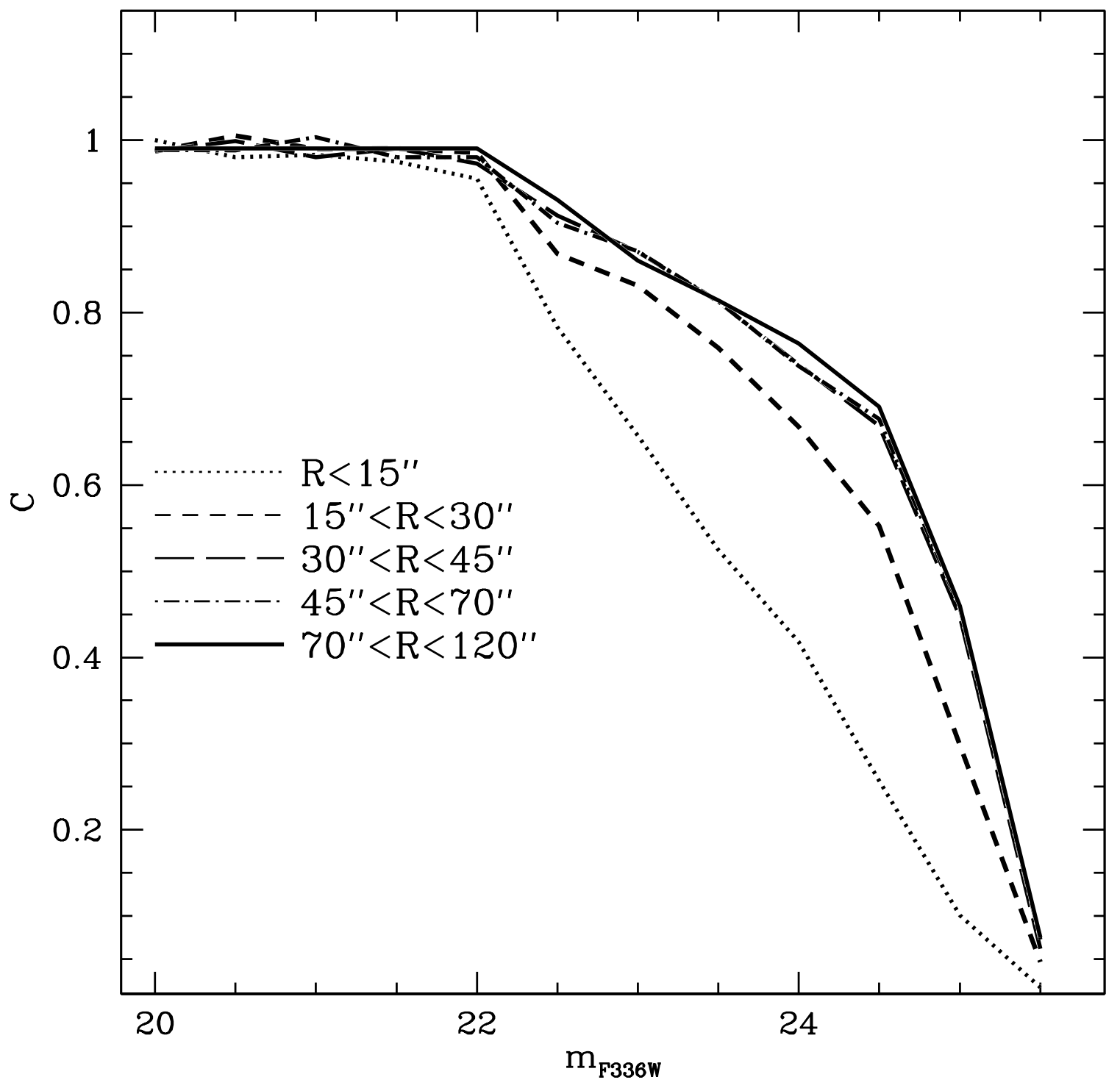

Fig. 4.- Photometric completeness $\mathrm{C}$ as a function of the $m_{\mathrm{F} 336 \mathrm{~W}}$ magnitude for different radial bins (see labels). 


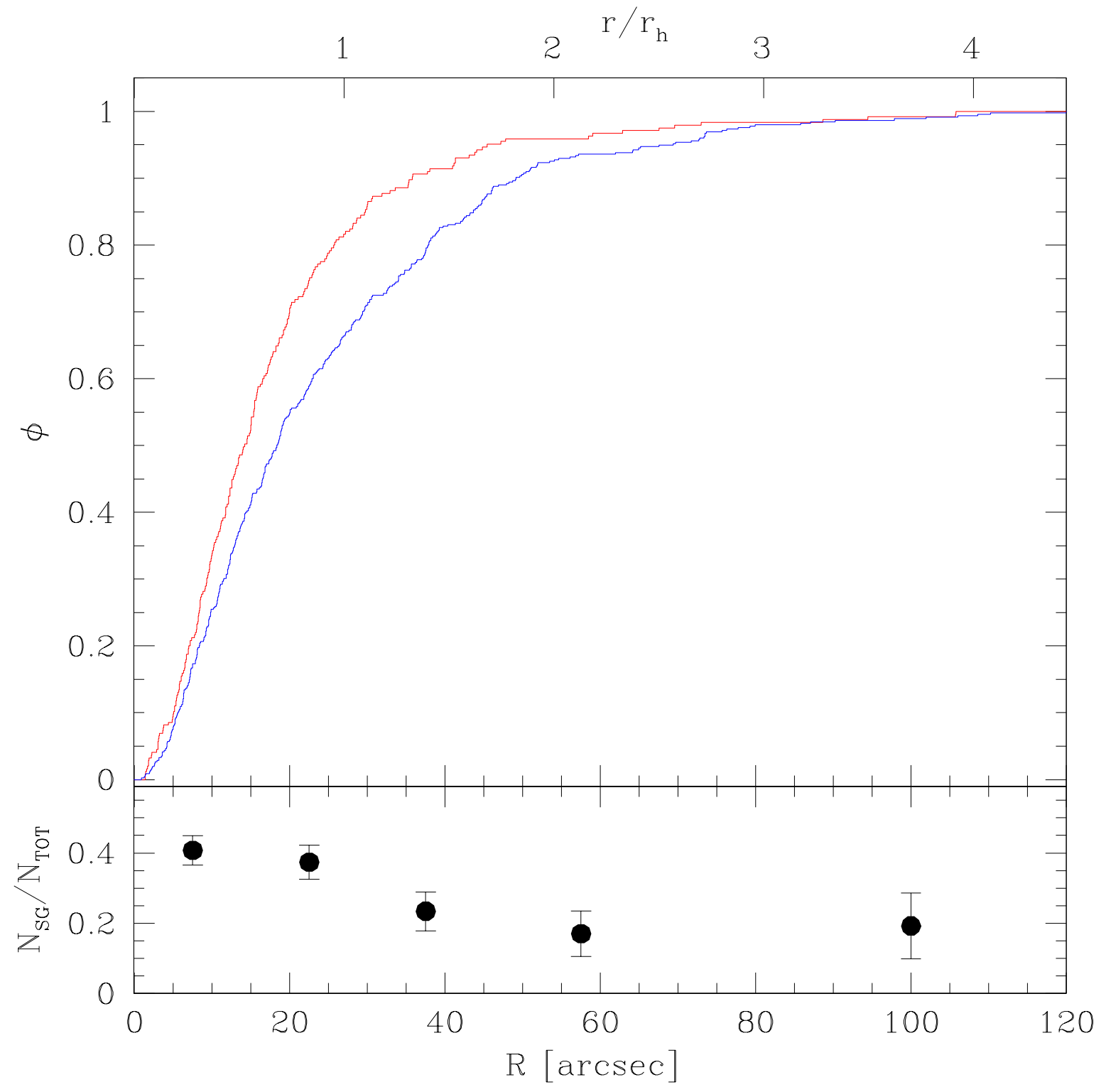

Fig. 5.- Upper panel: Cumulative radial distribution of FG (blue) and SG (red) stars within the WFC3 field of view selected as detailed in Section 3 as a function of the distance from the cluster center in arcsec and in units of $r_{h}$. Lower panel: $N_{\mathrm{SG}} / N_{\mathrm{TOT}}$ as a function of the distance from the cluster center. 

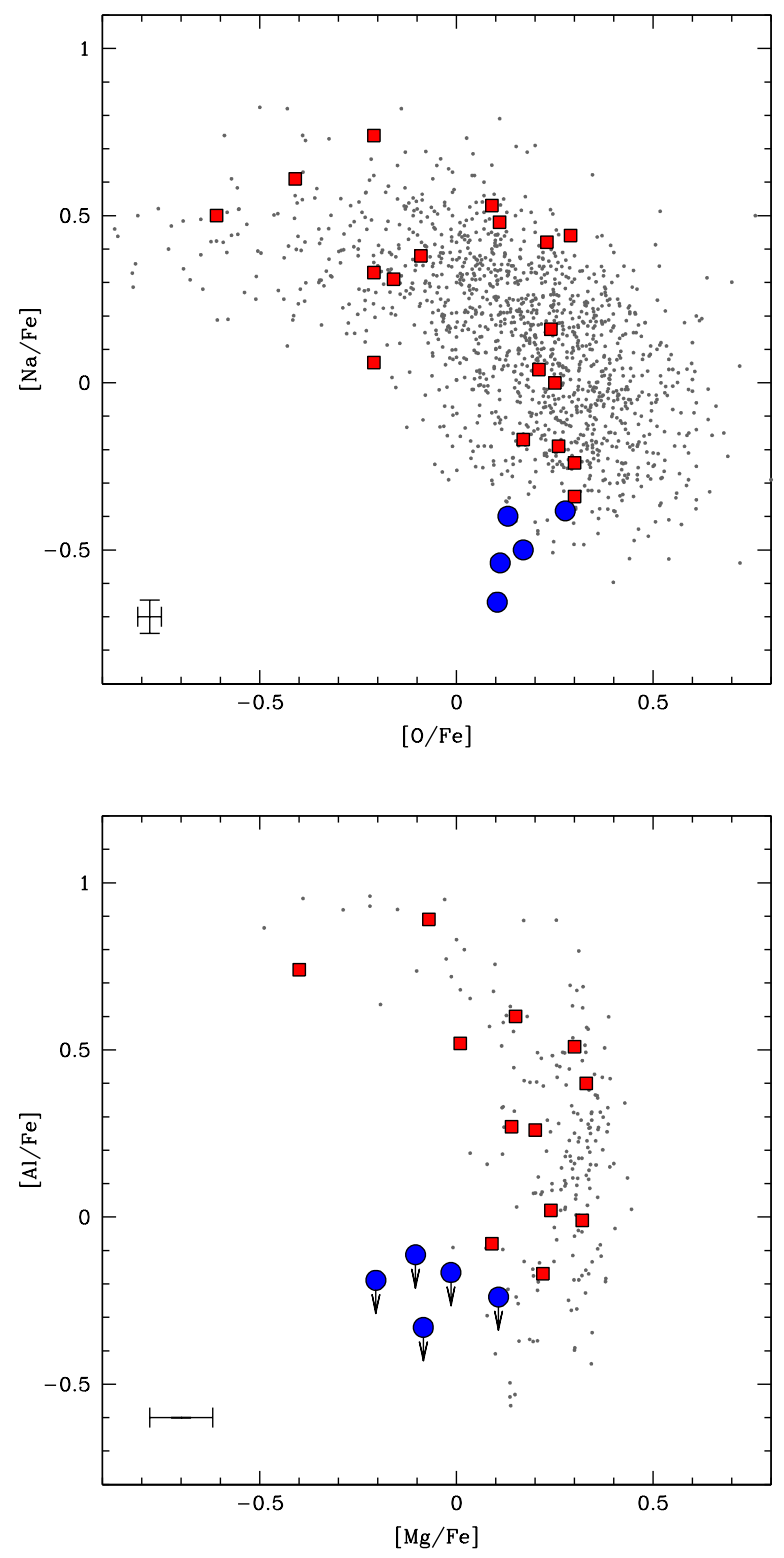

Fig. 6. - $[\mathrm{O} / \mathrm{Fe}]-[\mathrm{Na} / \mathrm{Fe}]$ (top panel) and $[\mathrm{Mg} / \mathrm{Fe}]-[\mathrm{Al} / \mathrm{Fe}]$ (bottom panel) diagrams. The blue solid circles are the target stars in NGC 121. For Al only upper limits have been derived. Red symbols mark stars in old LMC GCs from Mucciarelli et al. (2009a). Gray dots mark the values for stars in Galactic GCs (Carretta et al. 2009a,b). 


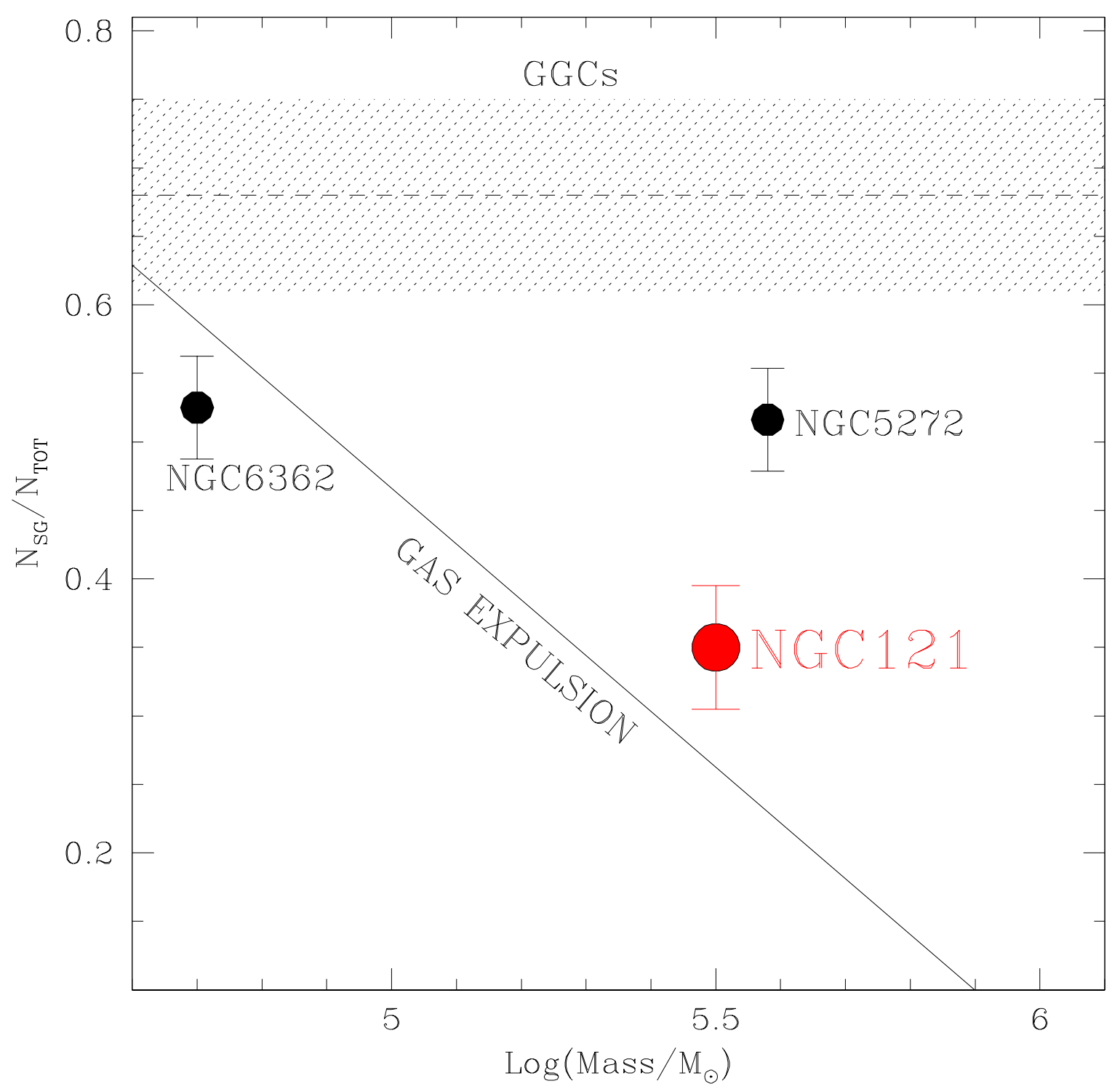

Fig. 7.- Fraction of SG stars as a function of GC mass. The values for NGC 121 (this work) and for NGC 6362 (Dalessandro et al. 2014a) and NGC 5272 (Massari et al. 2016) inferred from photometric indicators are marked as big dots. The horizontal dashed line marks the number ratio mean value and the dashed area the $1 \sigma$ region for Galactic GCs, as estimated by Bastian \& Lardo (2015) using a collection of spectroscopic and photometric values available in the literature. The solid line is the prediction by Khalaj \& Baumgardt (2015) for gas expulsion driven mass-loss. 